\title{
Lost in Translation: Reflecting on a Model to Reduce Translation and Interpretation Bias
}

\author{
Pamela Kirkpatrick ${ }^{*}, 1$ and Edwin van Teijlingen ${ }^{2,3}$
}

\author{
${ }^{I}$ The Robert Gordon University, The school of Nursing and Midwifery, Garthdee Road, Aberdeen, AB10 7QG, Scotland, UK \\ ${ }^{2}$ School of Health \& Social Care, Royal London House, Christchurch Road, Bournemouth University, Bournemouth \\ BU1 3LT, UK \\ ${ }^{3}$ Manmohan Memorial Institute of Health Sciences, Purbanchal University, Kathmandu, Nepal
}

\begin{abstract}
Aim: This paper reflects on the language and translation challenges faced and interventions used whilst undertaking cross-cultural public health research in Nepal using translators.

Background: The growth in cross-cultural studies and international research highlights the use of translators and the associated challenges for researchers with regard to cultural and linguistic issues when collecting data in one language and analysing and reporting in another. The specific challenges when using translators are frequently overlooked in the research literature and translators and the interpretation processes omitted from the research methodology and/or discussion.

Methods: The experiences and challenges for an English-speaking nurse researcher working with a team of translators in a cross-cultural study on the sexual and reproductive health (SRH) of young people in Nepal, in 2006, is explored in this paper. The paper employs a case study approach.

The Study: Data were collected over four weeks observing the settings, and the SRH knowledge and practices of participants in two villages in Kathmandu Valley were identified using a questionnaire, which was administered as a structured interview, and from the nurse researcher's field notes. This provided information on the translation and interpretation events. Stages in the research process where error could occur are identified in the researchers model and the interventions taken to minimise these are discussed which clarify perspectives and opinions when researching in the field.

Findings and Conclusion: Reflecting on translation and interpretation challenges during the data collection process in a language which the nurse researcher does not speak, can help reduce (or avoid) potential mistakes and error. The paper reflects on a model of translation processes which emerged during fieldwork which helps understand what events happened and what actions were taken to obtain the most accurate data. Using a model such as this for cross cultural fieldwork may be useful for both novice and established researchers.
\end{abstract}

\section{INTRODUCTION}

Cross-cultural working and researching are becoming increasingly popular and international research is now widespread [1]. In an international setting, the conduct of research poses a number of special methodological challenges for the researcher, particularly in the establishment and maintenance of rigor if she has to rely on others for collecting, translating and interpreting various types of data [2]. The challenge to achieving rigor is much greater due to complexities of working with different cultures, values, belief systems and languages [3].

For example, Hunt and Bhopal [4] note that when researching ethnic groups we must be cognisant of the target population's values, beliefs and customs before designing and implementing a research project and that the translators

*Address correspondence to this author at the Robert Gordon University, The school of Nursing and Midwifery, Garthdee Road, Aberdeen, AB10 7QG; Tel: 01224 262957; E-mail: p.kirkpatrick@rgu.ac.uk should be at the core of decision making in relation to all processes involved [5]. They and others also contend that collecting data across languages must not be considered an obstacle but a challenge [6] and that working with translators allows researchers (in nursing and public health-related disciplines) to study in new ways and often in areas and locations not previously studied. This challenge, however, is multifaceted and complex.

Wallin and Ahlström make the distinction between translation and interpretation [7]. Translators change written material and information from one language into another whilst interpreters work mainly by speech, translating back and forth between two languages. Recognising the influence of translation and subsequent interpretation on the data and the validity of the final outcomes of the research is vital because, as Temple and Young [3 p171] suggest:

\footnotetext{
"The translator always makes her mark on the research, whether this is acknowledged or not....."
} 
Birbili states that when we collect data in one language and subsequently present the findings in another, the translators involved must make translation-related decisions [1]. These decisions, she contends, have a direct impact on the research validity and are directly related to the autobiography of translators, the linguistic competence of the translators, the translators' knowledge of the people under study and the circumstances in which the translation takes place [1 p.1]. Beck and colleagues also note that the translators' cultural experience, knowledge and qualifications influence the quality of the translations [8]. To assume that translators are merely transmitters of neutral messages is likely to inhibit access to understanding the translation process and the emerging data [9]. Researchers must be cognisant of the translators' 'material circumstances' relating to their perceived power and, to an extent, expertise over the target group [9].

Meleis offers us a number of criteria as a guide to developing the necessary culturally competent knowledge [10]. This includes communication, where the translator must understand the subtleties and variations inherent in the target language and also demonstrate evidence of trust building in an attempt to uncover the population's experiences through voluntary disclosure in an authentic and understandable way. Most important is the notion of power and that the differentials be reduced or, at the very least, acknowledged and recorded. For example, power, in terms of age, race, gender and knowledge can differ greatly between the researcher, the translators and the participants. Whilst efforts may be made to demonstrate more horizontal relationships e.g. in shared decision making with translators, this is not often possible with participants. The research team should also make efforts to decrease the vertical power structure in order to gain better sharing of true information when these power hierarchies are minimised [10].

Beck et al. [8] indicate that the detail, description and discussion of the characteristics of the translators and the processes translators are involved in should be given attention in the research methodology and the researcher should document what has been done to reduce power differences [10]. This is further endorsed by Squires [11] who suggests that key strategies for tackling methodological challenges when using translators includes 'maintaining conceptual equivalence', 'translator credentials' and the translator's role in the process. Some writers suggest the translator be elevated to co-researcher and involved in all stages of the research process in depth [9] as it is argued that translating and interpreting research data is as much about social and cultural perspective as it is about language [12].

A word or phrase spoken or written in one language may mean one thing to one person but offer a completely different meaning to different people [13]. Where lexical equivalence is not possible, Birbili [1] suggests that 'conceptual equivalence' is sought. Conceptual equivalence or 'comparability of meaning' is not a problem free ideal as the concept itself may not exist in the target language and culture. More recently a move has been made towards discussing and debating in a reflexive way with the translators the decisions made in the translation process [3]. However, preserving the conceptual equivalence of a participant's responses during participant interview is the most crucial aspect of mediating translation issues during a study [9]. Using a Dutch example (the second author's first language) if someone in the Netherlands says "Hij staat met de mond vol tanden" the translation literally is, "He stood with a mouthful of teeth", but the translated meaning should be, "He could not speak", in the sense of "He did not know what to say". We would expect a competent translator to not to use the literal translation, but the one that better reflects the intended meaning.

Criticism has been directed at the apparent failure of researchers to consider the vital role that translators play in translation and interpretation and the implications of not introducing them into the communication loop during the research process $[1,3,9,12,14]$. Using translators for translation and interpretation activities in research inappropriately and/or inconsistently threatens the robustness of the data collected and the validity of the outcomes [11]. Translators' various levels of invisibility and apparent insignificance in research studies have been documented in the literature.

In a recent systematic review of qualitative cross-cultural studies using interpreters Wallin and Ahlstrom found some evidence of visibility of interpreters in four out of thirteen studies and little information about the extent of their involvement in just under half [7]. Some referred to the interpreters 'knowing the aim of the study' and others being 'key informants on the culture' and being 'briefed about the interview'; some translators only transcribed interviews. Rarely were the translators' credentials considered or reported by the researcher [7].

As translators have a major role in the knowledge acquisition and data collection process, their involvement needs to be transparent and the power relationships and their potential influence on the outcomes must be reported [3]. Closer attention therefore needs to be paid to the effect a translator may have on the research process and its outcomes [6]. Murray and Wynne suggest making the translator's work more visible during the research process particularly when conducting interviews [15]. They specify that the desired transparency requires the researcher to make the translators' and interpreters' specific roles, involvement in and decision making explicit within research documentation and write up.

\section{BACKGROUND TO OUR CASE STUDY}

One could argue that in structured interviews (i.e. questionnaire type interviews) the dialogue is more predictable than for example that in qualitative studies where the lines of questioning may vary and change during the interview and that less attention to the translation issues is needed [6]. However, in a large quantitative study such as ours, where both the translation and interpretation 'events' are numerous, we could argue that the need is just as great for planning, monitoring, documenting and evaluating translation challenges as part of the ongoing reflexive approach to data collection and interpretation. Though there is a growing body of literature relating to translation and interpretation challenges in both qualitative and quantitative studies this has not, to date, been particularly helpful in proactively guiding the researcher in the field through potential translation pitfalls [2]. 
This case study details the research experiences, processes and challenges encountered by the first author during fieldwork in Nepal in 2006. The fieldwork covered a four-week quantitative public health study using a team of translators for designing, collecting and interpreting sexual and reproductive health data. This was carried out as part of the first author's M.Sc. dissertation and she joined an established team of public health workers in Nepal from the University of Aberdeen. Funding was thereafter available for SRH interventions to be implemented to help improve the SRH of young people on completion of the data collection. The paper aims to presents a model that the first author formulated during the data collection period in Nepal as the challenges emerged. It identifies the specific stages where inaccuracy and bias could be introduced impacting on both the research process and its findings. Thus illuminates specific areas where translation and interpretation occurred as part of this process. From highlighting these stages we are able to document and explain the specific steps taken to minimise bias where possible, in both a planned pro-active and reflexive way.

\section{METHODS}

The case study methodology focuses on individual 'cases' to explore practice and the context in which practice is examined has relevance on our understanding of it. Thus we are able to closely examine the research processes and the research practice [16]. This single case allows us to closely examine the dynamics of the main communicators involved in a cross-cultural study: the nurse researcher, the translators and the participants [6]. Through the use of a model (Fig. 1), the authors highlight stages in the data generation process where errors could be made during translation and interpretation events and shows how the researcher and the team adapted and developed processes to ensure data collection was as accurate as possible. The translation events model will be discussed once the context for the study has been explained.

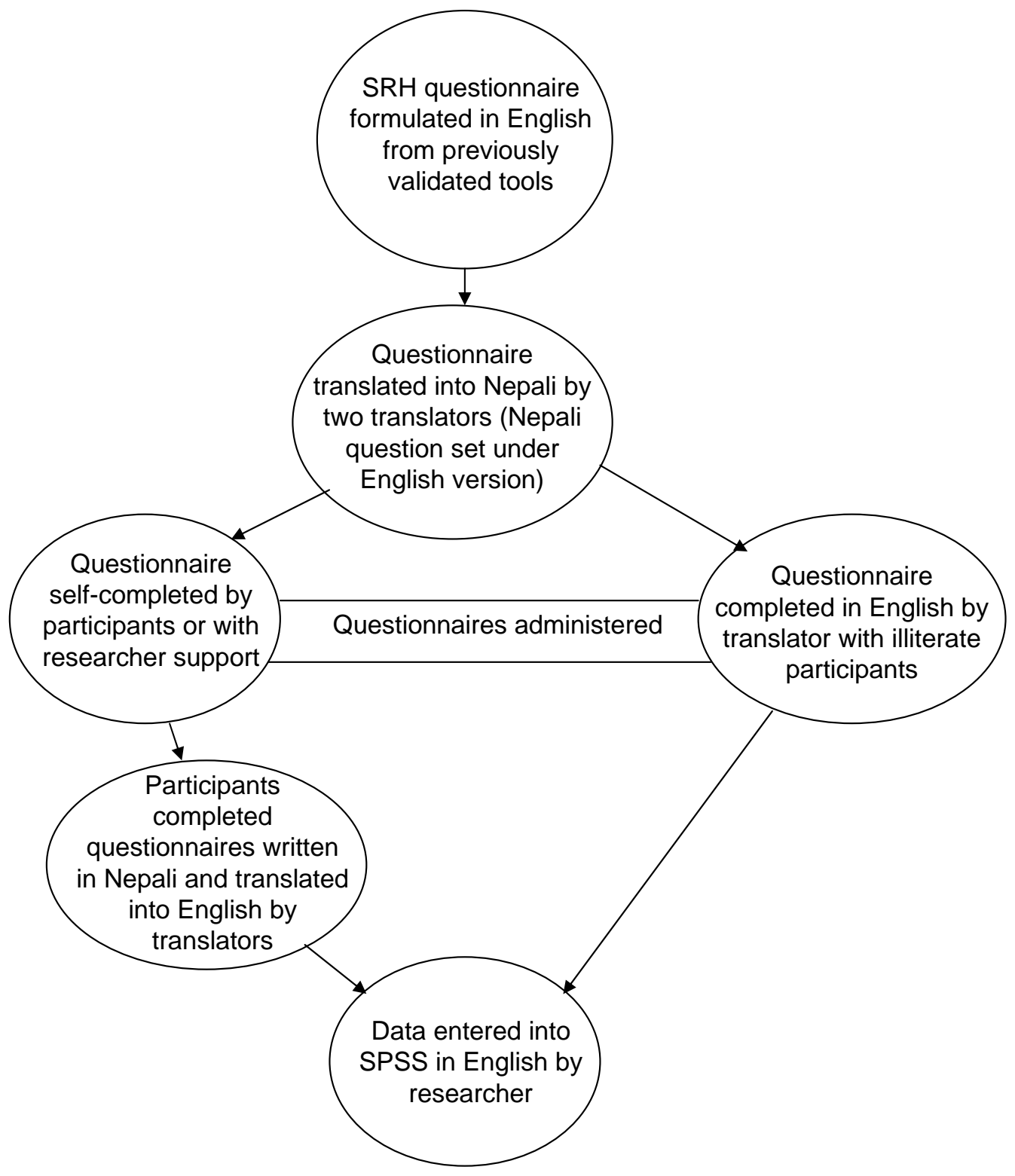

Fig. (1). Model of interpretation and translation process. 


\section{ETHICS}

Ethical approval was granted by the Nepal Health Research Council. Local support for the study was also given by the previous Village Development Committee (VDC) Chairman and members of the local community who were keen to participate in the study which they considered relevant to their health needs. The main issues of ethical concern centred on informed consent for young people and privacy and confidentiality. Informed and written consent was sought with a briefing statement for participants to read (and sometimes their guardian e.g. mother, teacher) or it being read to them before signing to participate or annotating with an ' $x$ '. The briefing statement also informed them of the private nature of the data collection and the anonymous and confidential nature of the collection, storage and reporting of it.

\section{OUR CASE STUDY}

The research study investigated the sexual and reproductive health (SRH) of males and females aged 13-24 in two neighbouring villages in Kathmandu Valley. The area is twenty kilometers from the capital city Kathmandu but it is relatively underdeveloped due to poor transport infrastructure, difficult terrain and constraints arising from national conflict. The study required the completion of an individual questionnaire by each participant to obtain information on his/her SRH. The focus of this research study was the SRH questionnaire: the purpose of this paper is to illuminate and consider the translation events and the potential for the translators' effects on the process of research. In order to ensure that the questions in the study were culturally appropriate and that they were sensitive to the participant's beliefs and values, the questionnaire design was closely based on two validated SRH questionnaires used previously in rural and urban Nepal $[17,18]$.

A convenience sampling method was used and participants were recruited through purposive, snowballing and opportunistic methods. This was done because there was very little relevant or accurate data about the target population in terms of demographic detail including population numbers, ages and gender. The most recent attempt to measure the demographics had been in the 2001 Census which was widely reported to be inaccurate since there had been a dramatic rise in the movement of large groups across geographical areas and countries in search of work.

The questionnaire consisted of 39 structured questions including multiple response, forced choice, closed questions and Likert scale selection questions. Two unstructured, free response questions on service availability and barriers to using the services concluded the questionnaire. Cognisance was given to the importance of how closely the wording in the questionnaire matched the 'meaning' of what was being asked rather than how closely it matched the 'words'. In any case, the Nepali vocabulary regarding sexual and reproductive health is limited compared to the English vocabulary and lexical matching is virtually impossible.

The nurse researcher was a 45-year-old white female who speaks English and has limited understanding of Nepali whilst the majority of the participants spoke Nepali only (with approximately $30 \%$ speaking English with variable fluency). The researcher was a Novice to undertaking primary research and also to working in a different culture to her own. The researcher underwent a week long induction programme on Nepal in her native country prior to embarking on the study to help gain some understanding of the culture and language. Gaining accurate meaning from the questionnaires, even with the support of a team of translators was challenging as the translation and interpretation process was complex and involved a number of steps, as outlined in the researcher's model (Fig. 1).

Given the large sample size required by the study (approximately $\mathrm{n}=250$ ) a team of translators was employed to finalise and translate the questionnaire into Nepali and help administer it in the villages. Five Nepali's were recruited in Nepal by the main translating coordinator. He was skilled in research and data collection in Nepal and had prior experience as a translator. He was educated to Masters Level in Public Health. Some of the translators were his colleagues and one a family member of a colleague, all having an excellent knowledge of Nepali culture. Two were female, one of them a fellow MSc student undertaking maternal and child research in the same villages. All had excellent written and spoken English and travelled daily to the study area from Kathmandu City. They all had graduate qualifications (most had a Master degree) though none were formally trained as translators. Two had previous interviewing and questionnaire administration experience in health research in Nepal. The interpreters appeared 'well shod', dressed impeccably in formal national dress or trousers, shirt and jacket and spoke with an educated highcaste accent. All translators received payment for the work they did. Table 1 illustrates the characteristics and the significant differences between the translators, researcher and participants. Again the power differentials are considerable here; however, translators required to have the ability to work between the researcher (and the study requirements) and the participants to generate the data.

In the days preceding data collection the team met frequently to discuss the practicalities and processes of data collection. Following recommended research practice we conducted a pilot study [19]. This indicated that we needed to reword, reorder and simplify some of the questions and improve the so-called filter questions. Due to the sensitive nature of some questions only male translators would interview male participants and females would interview females participants. This is common practice in research on sexual health $[20,21]$. This was to allow for 'gender and cultural sensitivity' and in the hope it would encourage more accurate and truthful disclosure. A point reinforced by Meleis as being vital for culturally competent research [10]. Translators were requested to ask the questions in the order presented and as worded in the questionnaire, indicating the participant's specific responses in pen on the questionnaire and for the open-ended questions to write down the spoken words verbatim. The pilot study involved ten illiterate individuals from outside the target communities. The pilot questionnaire revealed a high reliability of conceptual agreement though it provided no indication of how literate participants would mange the questionnaire. When the questionnaires were finalised each question was reworked and reworded to ensure the language used was linguistically 
Table 1. Characteristics of Nurse Researcher, Translators \& Participants

\begin{tabular}{|c|c|c|}
\hline Researcher & Translators & Participants \\
\hline \hline Female & Male/Female & Aged 13-24 \\
\hline Age 45 & Aged 27-38 & Married/Unmarried \\
\hline Married & Married/Unmarried & Children/No children \\
\hline Children & Children/No children \\
\hline MSc student/Lecturer in Nursing & Honours/Masters Degrees & No formal education/Masters Degree \\
\hline Non-poor & Non-poor & Mainly Poor \\
\hline Christian & Hindu & Nepali \\
\hline British & Nepali & Unknown experience of research/ Unlikely \\
\hline $\begin{array}{c}\text { No experience working with interpreter or field } \\
\text { research }\end{array}$ & $\begin{array}{c}\text { No experience working as translator/ some } \\
\text { experience working in research }\end{array}$ \\
\hline
\end{tabular}

and culturally appropriate. This was done by collaborating with the local seniors to check language and concept appropriateness.

The agreed process for administering the questionnaires was that participants could self-complete if literate or if illiterate a translator would conduct an interview with them and complete the questionnaire. Questionnaires were written in both Nepali and English and if participants were selfcompleting, they could read the questionnaire in Nepali/English and write their responses (where appropriate) in Nepali/English. Questionnaire completion was kept anonymous and confidential and where it was possible to administer a number of questionnaires at one time such as in a school setting, they were administered under examination conditions adhering to silence and privacy. A total of 253 questionnaires were collected and seven people refused to participate in the study.

\section{MODEL}

The researcher developed a model during the data collection process in order to visualise the specific stages of the research process where bias could be introduced to the data and information being collected. Biases in the translation and interpretation process were considered to be lack of conceptual equivalence, misunderstanding of language and misinterpretation of meaning. The model highlights the points where translation may be subjective and open to bias from each participant, translator or researcher. At each stage of the study/questionnaire design and research process challenges to the integrity of the meaning between the languages and culture existed and efforts were made to ensure these were minimised or removed where possible. Examples of these are now detailed.

The questionnaire was set in English and was translated by two translators in order to have the Nepali version of the questions positioned directly under the English version. The translators worked in partnership to complete the English to Nepali translation and both were unfamiliar with the questionnaire. Initially there was a disagreement between translators regarding the wording of one of the finalised questions in the questionnaire. Both translators felt the question asked something significantly different. This was discussed with the wider team of translators and researcher during meetings and but no consensus could be gained on the meaning or how best to word the question. Therefore, we decided to frame the question as presented in two previously validated tools used in Kathmandu for SRH and felt it did not require back translation from Nepali to English as a checking mechanism.

Once the daily data collection was completed the whole research team met at an arranged point weather permitting, and sifted through the completed questionnaires to check the data collected. Where self-completers completed in Nepali the translators would translate their entries into English and would discuss this with the researcher. If there were queries regarding the entries we would discuss these as a group.

For translator-completed questionnaires the translators were encouraged to record responses verbatim. Interruption during the interviews by anyone in the vicinity was discouraged as it was felt this would intrude on privacy and confidentiality and break the flow of the interview. The researcher was present during the interviews, where possible, observing questioning patterns, communication behaviours and interactions between translators and participants. At times however, the researcher might witness the five translators completing questionnaires with five participants and therefore close observation for all practice was not possible.

Frequently, the translators engaged in a 'warming up period' before the questionnaire questions were asked. During the process of gaining consent for participation translators sometimes took up to ten minutes to have a general chat with participants before starting. A period of discussion pre-interview can help build the relationship and promote trust and rapport between the interviewer and interviewee and may lead to greater disclosure [22]. Obtaining the most accurate data possible is dependent on the development of this rapport though people may not and do not have to reveal personal and intimate details about themselves [10, 22].

One question consisted of only eight words but took translators three to four sentences to ask. When the researcher questioned this the response was that the translators were asking the question as worded in the questionnaire but had to clarify the concept further for the 
participant. This raised concerns about the expression of the question and whether, in fact, conceptual equivalence had been achieved in all questions at the questionnaire formulation and translation stage. Translators were requested to keep the interviews as close to the questionnaire wording as possible as soon as the interview had begun whilst understanding the need for rapport building at the beginning.

An instance of deviation from the accepted interview process was the apparent giving of advice. It appeared to the researcher that occasionally the translator would offer health advice in response to participants' questions during the administration of the questionnaire. This posed the risk that the translator was being seen as a superior source of health knowledge and was therefore elevated further in position and power. Concerns here are linked to the issue of material position with the translator taking on a role of knowledge and expertise. If this occurred, it might have encouraged participants to answer what they thought the translator would expect them to say rather than provide the truth. The researcher requested that the translators wait until the questionnaire was complete before engaging with participants in health and health care related discussion.

Even though all but one of the translated questions gained agreement of 'concept' between translators, it became evident to the researcher during coding that one question did not appear to translate directly. When asking if participants had ever had a 'one night stand' three married women answered yes, although they claimed to have had only one sexual partner ever. These responses were found to be from illiterate women who had a translator complete the questionnaire for them. The translator was not expected to follow the responses through for logic therefore there was no way of affirming either response. This confirmed another anomaly in the translation. It is possible that this concept translated conceptually for them as 'having sexual intercourse once in a night' or even 'sexual intercourse on the first night of marriage'. These issues are culturally sensitive and many people may not want to talk about them or have not discussed or even heard them talked about in the Nepali language. It was noted by the research team this was a particularly difficult group of people to study because of the many instances where some of the language used and the meanings have not yet reached such remote and underdeveloped areas and had to be explained to participants.

When the study was complete one translator checked the translations from $10 \%$ of the free response questions for five participants who had completed the questionnaire by themselves who had recorded their responses in Nepali. This revealed a good inter-rater reliability supporting the robustness of the translation process.

A difficulty here lies with the notion of the translator having to act as a bridge across the cultural, linguistic and age-related gaps that are inevitable given the differences between the nurse researcher, translators and the target audience. Table 1 illustrates these main differences. The translator needs a level of freedom to enhance truthful reporting but on another level a systematic approach is needed. It may have been relevant to try to select translators who more closely matched to the participants in more of these respects.
Overall the model has been useful helping the researcher to visualise areas and stages where challenges to the acquisition of robust data may occur. Not all challenging areas were predictable from the outset but many potential pitfalls were avoided and some aspects adapted to help minimise the risk. The above examples are a small representation of the many challenges faced in this study and serve to illustrate the need for clarity, communication, teamwork and reducing error.

\section{DISCUSSION}

This paper has argued that many challenges are faced when carrying out research in a cross-cultural setting using translators. These unique challenges are posed by various contextual and cultural factors. The examples from the case study have reinforced the need for greater communication and collaboration between the researcher and translators at all stages of questionnaire design, translation, and recruitment of participants, data collection, translation, transcription and analysis. The study has detailed the importance of ongoing meetings, discussion and a problem solving approach to translation issues during these phases. Whilst we did not reach the research ideal suggested by Temple and Young [3] that the translators reached the status of co-researcher, they did have considerable voice and to a large extent, equity in decision making as the study progressed. Since this MSc dissertation did not have the resources that appeared to have been available to Lopez and colleagues [23] who employed trained bilingual interviewers and then separate translators for the translation of the transcripts, the input of our translators was very important in our attempt to attain a high degree of cultural competence in the study.

As the translators were the cultural and linguistic experts the researcher needed to spend time building a good rapport and a close working relationship with them in order to achieve a sense of partnership which would be conducive to effectively and efficiently progressing the study. This process was a natural part of the relationship development when working with a group of individuals in an intense, time-bound situation like the one described. At times however, communication took a concerted effort to ensure the robustness of the data collection in this sensitive topic with young people.

Squires argues that researcher competence is reflected in the degree of attention and detail given to the translation and translator issues in the methodological discussion minimizing risk of translation error [11]. Researchers who demonstrate that they have systematically accounted for any aspect that may impact on the findings of their research enhance the credibility, dependability, transferability and confirmability of the findings [11]. For these reasons the researcher must be adaptable and reflexive to changing demands and challenges throughout a study.

There is a view that studies suitable for native English speakers are, with minimum adaptation, suitable for other cultural groups [5]. Problems emanating from this attitude lead to issues of cultural hegemony, lack of salience of content, the non-equivalence of concepts, assumptions about willingness to disclose certain types of information and failure to ensure that the phenomenon of interest is present in 
all target groups [5]. As all these components were among the scope of the wider study we utilised questionnaires previously validated in Nepal. Nonetheless, we still found concepts and phrases unknown or strange to the target group. For example the word 'Giprog' or 'sex disease' was the local way of expressing 'STIs' (sexually transmitted infections). This reinforces the need for meaning of words over form and the importance of considering not only the national cultures and values but the local ones too [7].

The researcher considers the development of the model during data collection to have been beneficial even though it was verbalised and not visualised with the translators. It provided a systematic way of visiting and revisiting crucial stages of action and interaction during the research and worked as a trigger for wide and thorough team communication in a pro-active way. The researcher considers that this approach promoted greater validity in the findings which resembled the findings from other studies.

The experiences documented here are far from generalisable but they do serve to demonstrate the need for more debate around research in such challenging areas. However, attention is needed to the wider implications of some elements suggested such as an increase in time and monetary resources at the design stage. Pitchforth and van Teijlingen note that practical advice needs to be developed to help researchers develop relationships with translators and the wider issues involved in researching in these contexts [6].

This study demonstrates the importance for researchers of the need for:

- Achievement of conceptual equivalence rather than equivalence of word form;

- Involvement of translators at all stages of study design, development \& enactment;

- Collaborative relationships where the translator is promoted to co-researcher status;

- Clear communication and ongoing dialogue among team members throughout the study;

- $\quad$ Explicit negotiating and problem solving and in a team context;

- $\quad$ Explicit reporting of translator input in all stages of the study with acknowledgement of any potential influence on the outcome;

- $\quad$ A research study customised model to visualise and help guide the field researcher in achieving robustness;

- Guidelines for best practice for the researcher working in an international and cross-cultural context.

\section{REFLECTION ON FIELDWORK EXPERIENCE}

In this study the researcher cited translation issues as a limitation of the study in her final M.Sc. report though she did not explain in great detail how translators were utilised or what their input into discussion and decision making during the data collection was. This was due to dissertation word constraints and researcher naivety.
The nurse researcher did not explicitly demonstrate the emergent model to all members of the translation team though all aspects and concerns were verbalised throughout the questionnaire design and data collection period with everyone involved. In part this was due to the emergent nature of the model and practicalities in the field dealing with paperwork and carrying materials. In future studies the researcher will be better equipped and more able to prepare for these methodological challenges by adopting strategies such as using the translation events model visually and more explicitly and working even more collaboratively with translators. Developing a guide for 'Best practice when working with translators in cross-cultural research' could potentially be a helpful tool for both novice and experienced field researchers.

\section{CONCLUSIONS}

Being aware of the possible pitfalls in translation and interpretation issues helps both researchers and interpreters minimise them and this process should be explicit in the planning and reporting of research rather than accidental. Translators' work on all levels of a study must be made visible and clearly reported.

An interpretation/translation events model, specifically designed by the researcher for their study, is potentially useful to detail and highlight stages where bias may be a threat to producing accurate data.

Researchers' must have a good understanding of the culture they are researching and be cognisant of issues for culturally competent practice. It may be helpful to develop best practice guidelines for field researchers to use when undertaking research studies in an international or crosscultural context. Whereby a cross-cultural context could as easily refer to research with Afghan asylum seekers in Aberdeen, Scotland as to studies of Nepalese migrant workers in Mumbai, India.

\section{ACKNOWLEDGEMENTS}

The authors would like to thank Dr Padam Simkhada at the Department of Public Health, University of Aberdeen; Dr Jane Stephens, director of The Green Tara Trust, UK; Mr Prakash Bhatta, Mr Sharada Wasti, Mr Rupesh Bhatta, Miss Sarala Adikhiri and Mrs Soluchana Dhakal for their work as translators. Grateful thanks also go to the young people from the villages who participated in the study and all others who helped during the process. Finally, we like to extend out thanks to the three anonymous reviewers for The Open Nursing Journal for comments on our initial submission.

Financial support for travel and accommodation costs for this study was received from the University of Aberdeen Small Grants Fund.

\section{AUTHOR'S CONTRIBUTION}

PK contributed to the questionnaire design, carried out the data collection, input and analysis for this study as part of her MSc in Health Services and Public Health Research (Public Health). EvT was a joint supervisor of this research project at the University of Aberdeen and participated in most stages of the study. Both authors have written several drafts and approved the final manuscript. 


\section{REFERENCES}

[1] Birbili M. Translating from one language to another. Soc Res Update. 2000; 3: [Online] [Accessed 10 $0^{\text {th }}$ Feb 2009]. Available from: www.soc.surrey.ac.uk/sru/SRU31/html.

[2] Mill JE, Ogilvie LD. Establishing methodological rigor in international qualitative nursing research: a case study from Ghana. J Adv Nurs 2003; 41: 80-7.

[3] Temple B, Young A. Qualitative research and translation dilemmas. Qual Res 2004; 4: 161-78.

[4] Hunt S, Bhopal R. Self Reports in research with non-English speakers. Br Med J 2003; 327: 352-3.

[5] Hanna L, Hunt S, Bhopal R. Insights from research on crosscultural validation of health related questionnaires: the role of bilingual project workers and lay participants. Curr Sociol 2008; 56: 115-31.

[6] Pitchforth E, van Teijlingen E. International public health research involving interpreters: a case study from Bangladesh. BMC Public Health 2005; 5: 71. [Accessed $10^{\text {th }}$ Feb 2009]. Available from: http://www.biomedcentral.com/content/pdf/1471-2458-5-71.pdf

[7] Wallin AM, Ahlström G. Cross-cultural interview studies using interpreters: systematic literature review. Integr Lit Rev Meta-Anal 2007; 55: 723-35

[8] Beck CT, Bernal H, Froman RD. Methods to document semantic equivalence of a translated scale. Res Nurs Health 2003; 26: 64-73.

[9] Larkin PJ, de Casterlé BD, Schotsmans P. Multilingual translation issues in qualitative research: reflections on a metaphorical process. Qual Health Res 2007; 17: 468-76.

[10] Meleis AI. Culturally competent scholarship: substance and rigor. Adv Nurs Sci 1996; 19: 1-16.

[11] Squires A. Methodological challenges in cross-Language qualitative research: a research review. Int J Nurs Stud 2008; 55 : 265-73.
[12] Temple B. Crossed wires: interpreters, translators and bilingual workers in cross-language research. Qual Health Res 2002; 12: 844-54.

[13] Eboh WO, Pitchforth E, van Teijlingen E. Lost words: research via translation. RCM Midwives J 2007; 10: 374-7.

[14] Temple B, Edwards R. Interpreters/translators and cross language research: reflexivity and border crossings. Int $\mathrm{J}$ Qual Methods 2002; 1: 1-22.

[15] Murray CD, Wynne J. Researching community, work and family with an interpreter. Commun Work Fam 2001; 4: 151-71.

[16] Gerrish K, Lacey A, Eds. Research process in nursing. $5^{\text {th }}$ ed. Oxford: Blackwell Publishing 2005.

[17] Cleland J, Ingham R, Stone N. UNDP/UNFPA/WHO/World Bank Special Programme of research, Development and Training in Human reproduction. Asking young people about sexual and reproductive behaviours: Illustrative Core Instruments. 2000; [Online]. [Accessed 20 ${ }^{\text {th }}$ Feb 2009]. Available from: http:// www.who.int/reproductive-health/adolescent/questionnaire.html

[18] Mahat G, Scoloveno MA. HIV/AIDS Knowledge, attitudes and beliefs among Nepalese adolescents. J Adv Nurs 2006; 53: 583-90.

[19] Teijlingen van E, Hundley V. Pilot studies in family planning and reproductive health care. J Fam Plann Reprod Health Care 2005; 31:219-21.

[20] Halstead M, Waite S. 'Worlds Apart' The sexual values of boys and girls. Educ Health 2002; 20: 17-23.

[21] Teijlingen van E, Reid J, Shucksmith J, et al. Embarrassment as a key emotion in young people talking about sexual health, Sociol Res Online 2007; 12: [Online] [Accessed 29 ${ }^{\text {th }}$ Jan 2009]. Available from http://www.socresonline.org.uk/12/2/van teijling en.html

[22] Nunkoosing K. The Problems with interviews. Qual Health Res 2005; 15: 698-706.

[23] Lopez GI, Figueroa M, Connor SE, Maliski SL. Translation barriers in conducting qualitative research with Spanish speakers. Qual Health Res 2008; 18: 1729-37.

Received: April 15, 2009

Revised: May 22, 2009

Accepted: May 22, 2009

(C) Kirkpatrick and van Teijlingen; Licensee Bentham Open .

This is an open access article licensed under the terms of the Creative Commons Attribution Non-Commercial License (http: //creativecommons.org/licenses/by$\mathrm{nc} / 3.0 /$ ) which permits unrestricted, non-commercial use, distribution and reproduction in any medium, provided the work is properly cited. 